\title{
Discontinuous Galerkin spectral element method for solving population balance differential equations
}

\author{
Mahmoud Lotfi
}

\begin{abstract}
In this paper, we study the discontinuous Galerkin spectral element method for solving the population balance differential equation. We use the Legendre polynomials of order $k$ as test functions on each element. Calculate the matrices used by Gauss Quadrature integration and then compare the numerical results with the other methods.
\end{abstract}

Keywords-Discontinuous Galerkin, spectral element method, population balance differential equation, Legendre-Gauss Quadrature.

\section{INTRODUCTION}

$\mathbf{P}$ OPULATION balance differential equations (PBDEs) have been introduced in several branches of modern science, mainly in chemical Engineering such as crystallization from solution, emulsion polymerization, microbial populations, particle agglomeration, and residence time distributions in reactors [1], [2]. However, the population balance equations are rather complicated. Therefore, they are often analyzed in terms of the moments of the number density function. Some different numerical methods to solve PBDEs were presented. Singh and Ramkrishna in [3] used the method of weighted residual for solving the PBDEs. The method of rationalized Haar has been used to solve PBDEs [4]-[6]. Singh and Ramkrishna use the Vorobyevs method [7]. Randolph also used Adams-Moultion-Shell method to solve PBDEs [8]. Block-Pulse method are also use for numerical solution of these types of equations [9], Shifted Legender method [10] and Wavelet-Galerkin method [11] have been used to obtain approximation solution of population balance equations.

\section{A. A brief review on discontinuous Galerkin spectral element method}

The discontinuous Galerkin spectral element method is a method for converting a continuous problem to a discrete problem. The weak formulation of the method is used to represent piecewise polynomials that are discrete on the boundary of each element. Weak formulation is proposed in the following. We use the Legendre Gaussian Quadrator to compute integrals.

\section{B. Weak formulation}

Consider the differential equation below

$$
\begin{aligned}
& \frac{d u}{d x}=f, \\
& f \in R, \quad x \in R^{\geq 0} .
\end{aligned}
$$

Mahmoud Lotfi is with the Department of Applied Mathematics, University of Kurdistan, Sanandaj, IRAN

Email: lotfi.mahmud@gmail.com and m.lotfi@sci.uok.ac.ir
By multiplying the two sides of the above equation in the test function $v$ on the Euclidean space $\Omega=\left\{[a, b] \in R,\|\cdot\|_{2}\right\}$, and using inner product on $L^{2}$

$$
(u, v)=\int_{\Omega} u v d x
$$

we have

$$
\int_{\Omega} \frac{d u}{d x} v d x=\int_{\Omega} f v d x .
$$

Using integration by parts

$$
[u v]-\int_{\Omega} u \frac{d v}{d x} d x=\int_{\Omega} f v d x .
$$

The above equation is the weak form of the problem, and by solving it, the weak answer is obtained. This is not unique, since it is dependent on the test function and there is an infinite number of test functions available.

\section{Legendre-Gauss Quadrature}

Legendre-Gauss quadrature is a numerical integration method also called the Gaussian quadrature or Legendre quadrature. A Gaussian quadrature over the interval $[-1,1]$ with weighting function $W(\theta)=1$. The abscissas for quadrature order $N$ are given by the roots of the Legendre polynomials $\mathcal{L}_{n}(\theta)$

$$
\mathcal{L}_{N}(\theta)=2^{-N} \sum_{i=0}^{\left[\frac{N}{2}\right]}(-1)^{i}\left(\begin{array}{l}
N \\
i
\end{array}\right)\left(\begin{array}{l}
2 N-2 i \\
N
\end{array}\right) \theta^{N-2 i} .
$$

The weights are

$$
w_{i}=-\frac{\alpha_{N}}{\alpha_{N-1}} \frac{\beta_{N-1}}{\mathcal{L}_{N-1}\left(\theta_{i}\right) \mathcal{L}^{\prime}{ }_{N}\left(\theta_{i}\right)},
$$

where $\alpha_{N}$ is the coefficient of $\theta^{N}$ in $\mathcal{L}_{n}(\theta)$. For Legendre polynomials,

$$
\alpha_{N}=\frac{(2 N) !}{2^{N}(N !)^{2}},
$$

additionally [12],

$$
\beta_{N}=\int_{-1}^{1}\left[\mathcal{L}_{N}(\theta)\right]^{2} d \theta=\frac{2}{2 N+1} .
$$

The weights $w_{i}$ satisfy

$$
\sum_{i=1}^{N} w_{i}=2
$$

The error term is

$$
\text { Error }=\frac{2^{2 N+1}(N !)^{4}}{(2 n+1)[(2 N) !]^{3}} f^{(2 N)}(\xi) .
$$




\section{SOLVING THE POPULATION BALANCE DiFFERENTIAL EQUATION USING THE DISCONTINUOUS GALERKIN SPECTRAL ELEMENT METHOD}

We consider the population balance differential equation below

$$
\frac{d y(x)}{d x}+\left(1+\kappa x^{m}\right) y(x)=2^{m+1} \kappa x^{m} y(2 x), 0 \leq x \leq b,
$$

where $\kappa$ and $m$ are constants. The initial value $y(x)$ is also as follows

$$
y(0)=1 \text {. }
$$

Notice that on the right hand side of the equation (11) is a statement $y(2 x)$, and on the left hand is the phrase $y(x)$. It has appeared that it does not have an analytic solution [13][15], and so, approximate methods for solving the Eq. (11) are of particular importance. Various numerical methods for solving the population balance differential equation (11) are presented, including the Block-pulse functions [14], method of weighted residuals [15], wavelet-Galerkin [13], and the use of rationalized Haar functions [5].

From Figure 1, to solve Eq. (11) with discontinuous Galerkin spectral element method we put ( $N_{e}$ is number of elements)

$$
\begin{aligned}
& \Omega_{e}=\left(x_{e-1}, x_{e}\right), \quad e=1, \ldots, N_{e}, \\
& v_{+}^{n}=\lim _{s \rightarrow 0^{+}} v\left(x_{e}+s\right), \\
& v_{-}^{n}=\lim _{s \rightarrow 0^{-}} v\left(x_{e}+s\right) .
\end{aligned}
$$

So we have:

For each element $\Omega_{e}$, find $y_{h} \in P_{N}\left(\Omega_{e}\right)$, such that

$$
B_{e}\left(y_{h}, v_{h}\right)=L_{e}\left(v_{h}\right), \quad \forall v_{h} \in P_{N}\left(\Omega_{e}\right)
$$

where $P_{N}$ is a polynomial space of less than or equal to $N$ on $\Omega_{e}$, and

$$
\begin{aligned}
& B_{e}\left(y_{h}, v_{h}\right)=\int_{\Omega_{e}}\left(y_{h}(x) v^{\prime}{ }_{h}(x)+a(x) y_{h}(x) v_{h}(x)\right. \\
& \left.-b(x) y_{h}(2 x) v_{h}(x)\right) d x+y_{h_{+}^{e-1}} v_{h_{+}}^{e-1}, \\
& L_{e}\left(v_{h}\right)=y_{h-}^{e-1} v_{h+}^{e-1}, \\
& L_{e}\left(v_{h}\right)=y_{h-}^{e-1} v_{h+}^{e-1}, \\
& b(x)=2^{m+1} \kappa x^{m} .
\end{aligned}
$$

The initial condition will be $y_{h-}^{0}=1$. Note that $y_{h}$ and $v_{h}$ are polynomials of the maximum degree $N$ on $\Omega_{e}$, which makes them discrete on the boundaries.

We define the space $V$ as follows $V=\left\{v: v\right.$ is continuous on the intervals $\left.\Omega_{e}, e=1, \ldots, N_{e}\right\}$.

Let that $v(x) \in V$ is an arbitrary function and $y(x)$ is the exact solution of the Eq. (11). By multiplying Eq. (11) in $v$, we have

$$
y^{\prime}(x) v(x)+a(x) y(x) v(x)-b(x) y(2 x) v(x)=0 .
$$

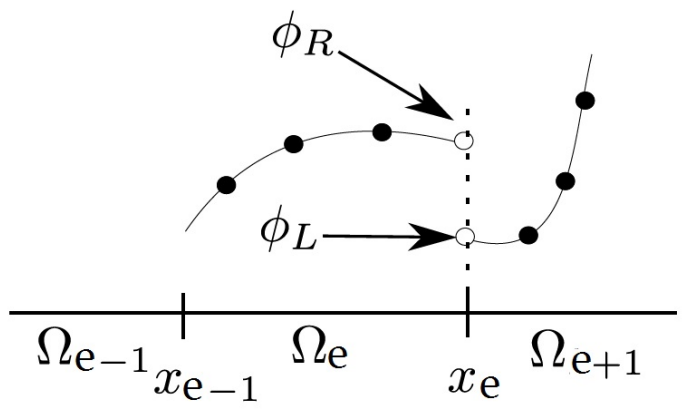

Fig. 1. Boundary values of subdomains

Now by integrating on the interval $(0, b)$ we have

$$
\begin{aligned}
& \sum_{e=1}^{N_{e}}\left[\int _ { \Omega _ { e } } \left(y^{\prime}(x) v(x)+a(x)\right.\right.y(x) v(x)-b(x) y(2 x) v(x)) d x \\
&\left.+\left(y_{+}^{e-1}-y_{-}^{e-1}\right) v_{+}^{e-1}\right]=0,
\end{aligned}
$$

and since $v$ is independent of the selection of subscripts, with integration by part we can get variational form of problem

$$
B_{e}(y, v)=L_{e}(v), \forall v \in V .
$$

By replacing $y$ with $y_{h} \in P_{N}\left(\Omega_{e}\right)$ and $v$ with $v_{h} \in P_{N}\left(\Omega_{e}\right)$ we have the discontinuous Galerkin spectral element method equation.

To calculate the integral $B(v, y)$, the subdomain $\Omega_{e}$ is first transferred to the domain $(-1,1)$ using the following mapping [16]

$$
\begin{aligned}
& x(t)=\frac{1}{2}\left[\left(x_{e}-x_{e-1}\right) t+\left(x_{e}+x_{e-1}\right)\right], \quad-1 \leq t \leq 1, \\
& t(x)=2 \frac{x-x_{e-1}}{x_{e}-x_{e-1}}-1, \quad x_{e-1} \leq x \leq x_{e} .
\end{aligned}
$$

So

$$
\begin{aligned}
& \int_{\Omega_{e}}\left(y^{\prime}(x) v(x)+a(x) y(x) v(x)-b(x) y(2 x) v(x)\right) d x \\
& =\int_{-1}^{1} y^{\prime}(t) v(t) d t+\frac{h}{2} \int_{-1}^{1}(a(t) y(t) v(t)-b(t) y(2 t) v(t)) d t,
\end{aligned}
$$

where $h=x_{e}-x_{e-1}$. The variational form of the problem, will also be as follows

Find $y_{h} \in P_{N}(-1,1)$ such that

$$
B_{e}\left(y_{h}, v_{h}\right)=L_{e}\left(v_{h}\right), \quad \forall v_{h} \in P_{q}(-1,1),
$$

where

$$
\begin{aligned}
& B_{e}\left(y_{h}, v_{h}\right)=\int_{-1}^{1} y^{\prime}{ }_{h}(t) v_{h}(t) d t \\
& \quad+\frac{h}{2} \int_{-1}^{1} a(t) y_{h}(t) v_{h}(t)-b(t) y_{h}(2 t) v_{h}(t) d t+y_{+}^{e-1} v_{+}^{e-1}, \\
& L_{e}\left(v_{h}\right)=y_{-}^{e-1} v_{+}^{e-1} .
\end{aligned}
$$




\section{A. Discontinuous Galerkin spectral element method of order} $k$

To solve the problem using discontinuous Galerkin spectral element method of order $k$, we define test functions as follows

$$
\begin{aligned}
\varphi_{j}(t)=\frac{1}{k(k+1) \mathcal{L}_{k}\left(t_{j}\right)} \frac{\left(t^{2}-1\right) \mathcal{L}^{\prime}{ }_{k}(t)}{t-t_{j}}, \\
0 \leq j \leq k,-1 \leq t \leq 1,
\end{aligned}
$$

where $\varphi_{j}$ is the $j^{\text {th }}$ Lagrange polynomial of order $k$ on the Gauss-Legendre-Lobatto points $\left\{t_{j}\right\}_{j=0}^{k}$ [17].

We put

$$
\begin{aligned}
& y(t)=\varphi_{0}(t) y_{+}^{e-1}+\sum_{j=1}^{k-1} y_{j} \varphi_{j}(t)+\varphi_{k}(t) y_{-}^{e}, \\
& y^{\prime}(t)=\varphi_{0}^{\prime}(t) y_{+}^{e-1}+\sum_{j=1}^{k-1} y_{j} \varphi_{j}^{\prime}(t)+\varphi^{\prime}{ }_{k}(t) y_{-}^{e}, \\
& v(t)=\varphi_{0}(t) v_{+}^{e-1}+\sum_{j=1}^{k-1} v_{j} \varphi_{j}(t)+\varphi_{k}(t) v_{-}^{e} .
\end{aligned}
$$

By replacing the above values in (14), the matrix form of the problem is obtained as follows

$$
\begin{gathered}
A Y=B \\
A_{1,1}=\int_{-1}^{1}\left[\varphi_{0}(t) \varphi_{0}^{\prime}(t)+\frac{h}{2} a(t) \varphi_{0}(t) \varphi_{0}(t)\right. \\
\left.-b(t) \varphi_{0}(2 t) \varphi_{0}(t)\right] d t+1, \\
A_{i, j}=\int_{-1}^{1}\left[\varphi_{i}(t) \varphi_{j}^{\prime}(t) d t+\frac{h}{2} a(t) \varphi_{i}(t) \varphi_{j}(t)\right. \\
\left.-b(t) \varphi_{i}(2 t) \varphi_{j}(t)\right] d t, i+j>2 k, \\
Y=\left[\begin{array}{l}
y_{+}^{e-1} \\
\vdots \\
y_{-}^{e}
\end{array}\right], \\
B=\left[\begin{array}{l}
y_{-}^{e-1} \\
0 \\
\vdots \\
0
\end{array}\right] .
\end{gathered}
$$

Now by zeroing top-diameter substrates of the matrix $A$, we have

$$
\left[\begin{array}{cccc}
0 & \cdots & 0 & a_{1, k+1} \\
\vdots & . & . & \vdots \\
0 & . & . & \vdots \\
a_{k+1,1} & . & \cdots & a_{k+1, k+1}
\end{array}\right]\left[\begin{array}{c}
y_{+}^{e-1} \\
\vdots \\
\vdots \\
y_{-}^{e}
\end{array}\right]=\left[\begin{array}{l}
y_{-}^{e-1} \\
0 \\
\vdots \\
0
\end{array}\right]
$$

So, the following recursive relation can be obtained

$$
y_{-}^{e}=\frac{1}{a_{1, k+1}} y_{-}^{e-1}
$$

\section{B. Calculation the matrix $A$}

The matrices of $A$ contain the following integrals

$$
\begin{aligned}
& S_{i, j}=\int_{-1}^{1} \varphi_{i}(t) \varphi^{\prime}{ }_{j}(t) d t \\
& M 1_{i, j}=\int_{-1}^{1} a(t) \varphi_{i}(t) \varphi_{j}(t) d t \\
& M 2_{i, j}=\int_{-1}^{1} b(t) \varphi_{i}(2 t) \varphi_{j}(t) d t
\end{aligned}
$$

1) Calculation the $S_{i, j}$ : Using Gauss Quadrature, we have

$$
S_{i j}=\sum_{p=1}^{k+1} \varphi_{i}\left(t_{p}\right) d_{j p} w_{p},
$$

where

$$
\begin{array}{ll}
d_{j p}=\left.\frac{d \varphi_{j}}{d t}\right|_{t_{p}}=\frac{L_{k}\left(t_{p}\right)}{L_{k}\left(t_{j}\right)} \frac{1}{t_{p}-t_{j}}, & (j \neq p), \\
d_{j j}=\left.\frac{d \varphi_{j}}{d t}\right|_{t_{j}}=\frac{L_{k}^{\prime}\left(t_{j}\right)}{2 L_{k}\left(t_{j}\right)}, \quad & (j=p),
\end{array}
$$

where $t_{p}$ are the roots of $\mathcal{L}_{k}^{\prime}(t)$, then for $j=2, \ldots, k$ we have $d_{j j}=0$, and

$$
\begin{aligned}
& d_{11}=\frac{L_{k}^{\prime}(-1)}{2 L_{k}(-1)}=-\frac{1}{4}(N(k+1)) \\
& d_{k+1, k+1}=\frac{L_{k}^{\prime}(1)}{2 L_{k}(1)}=\frac{1}{4}(N(k+1))
\end{aligned}
$$

Also, we have

$$
w_{p}=\frac{2}{(N(k+1))\left[L_{k}\left(t_{p}\right)\right]^{2}}, \quad 1 \leq p \leq k+1 .
$$

Definition 2.1: $A^{n \times n}$ is a pseudo-antisymmetric matrix which satisfies the below conditions:

1. $a_{11} \neq 0, a_{n n} \neq 0$,

2. for $i \neq 1$ and $i \neq n, a_{i, i}=0$,

3. for all $i$ and $j$ that $i \neq j, a_{i, j}=-a_{j, i}$.

Lemma 2.2: $S$ is pseudo-antisymmetric matrix.

Proof: By integration by part we have

$$
\begin{aligned}
S_{i j}= & \int_{-1}^{1} \varphi_{i}(t) \varphi_{j}^{\prime}(t) d t \\
& =\left.\varphi_{i}(t) \varphi_{j}(t)\right|_{-1} ^{1}-\int_{-1}^{1} \varphi_{i}^{\prime}(t) \varphi_{j}(t) d t .
\end{aligned}
$$

Now if $i \neq j, i, j \neq 1$ and $i, j \neq k+1$, then we have

$$
\varphi_{i}( \pm 1)=0, \quad \varphi_{j}( \pm 1)=0 .
$$

So for each $i \neq j, i, j \neq 1$ and $i, j \neq k+1$, we have

$$
\begin{aligned}
S_{i j}= & \int_{-1}^{1} \varphi_{i}(t) \varphi_{j}^{\prime}(t) d t= \\
& -\int_{-1}^{1} \varphi_{i}^{\prime}(t) \varphi_{j}(t) d t=S_{j i} .
\end{aligned}
$$

Then

$$
S_{i j}=-S_{j i} .
$$

For $i=j, i, j \neq 1$ and $i, j \neq k+1$

$$
S_{i i}=-S_{i i} \quad S_{i i}=-S_{i i} \quad \Rightarrow 2 S_{i i}=0,
$$

and so $S_{i i}=0$ 
The first and last value of the matrix are also calculated as follows

$$
\begin{gathered}
S_{11}=\varphi_{1}(1)^{2}-\varphi_{1}(-1)^{2}-S_{11} \Rightarrow S_{11}=\frac{\varphi_{1}(1)^{2}-\varphi_{1}(-1)^{2}}{2} \\
S_{k+1, k+1}=\varphi_{k+1}(1)^{2}-\varphi_{k+1}(-1)^{2}-S_{k+1, k+1} \\
\Rightarrow S_{k+1, k+1}=\frac{\varphi_{k+1}(1)^{2}-\varphi_{k+1}(-1)^{2}}{2} .
\end{gathered}
$$

According to the previous lemma, for the calculation of the matrix of the order $k+1, S$ is sufficient to calculation the $\frac{k(k+1)}{2}+2$ values in the matrix.

2) Calculation the $M 1_{i, j}$ : Since the $a(t)=1+\kappa t^{m}$, so we have

$$
\begin{gathered}
M 1_{i j}=\int_{-1}^{1} \varphi_{i}(t) \varphi_{j}(t) d t+k \int_{-1}^{1} t^{m} \varphi_{i}(t) \varphi_{j}(t) d t \\
=N 1_{i j}+N 2_{i j}
\end{gathered}
$$

$N 1$ is a mass matrix and its values are calculated as follows [18]

$$
N 1_{i j}=\delta_{i j} w_{i}
$$

And we use the Gauss quadrature to calculation $N 2$ directly

$$
N 2_{i j}=\sum_{p=1}^{k_{1}} t_{p}^{m} \varphi_{i}\left(t_{p}\right) \varphi_{j}\left(t_{p}\right) w_{p}
$$

Where $2 k_{1}+1 \geq 2 k+m$. According to the above equation, if we use polynomials of the same degree on each celement, the N2 matrix is the same on each element, and it is not necessary to recalculate it.

3) Calculation the $M 2_{i, j}: M 2$ is calculated using the Gauss quadrature as follows

$$
\int_{-1}^{1} b(t) \varphi_{i}(2 t) \varphi_{j}(t) d t=\sum_{p=1}^{k_{2}} b\left(t_{p}\right) \varphi_{i}\left(2 t_{p}\right) \varphi_{j}\left(t_{p}\right) w_{p}
$$

Where $k_{2}$ applies in the condition of optimal accuracy of the Gauss quadrature integral.

\section{NUMERICAL RESULTS}

Since there is no exact solution for the Eq. (11), we compare the results obtained from the method presented in the previous sections with the wavelet Galerkin [13] and the rationalized Haar functions [5]. We have solved the Eq. (11) with $\kappa=\frac{1}{162}$, $m=4$ and $b=6$ in this paper. Table I shows the results of DGSEM with two values of $k$ and results obtained of wavelet Galerkin and rationalized Haar functions. Figure 2 show graph of approximate solution with $k=4$ and $N_{e}=12$.

\section{CONCLUSiON}

Since population balance differential equation do not have the exact solution, and have a great deal of relevance in various sciences, it is important to find an approximate solution. For this reason, several numerical methods are proposed for the approximate solution of these equations. We have used the discontinuous Galerkin spectral element method to approximate
TABLE I

COMPARE THE NUMERICAL RESULTS WITH $N_{e}=12$

\begin{tabular}{|r|l|l|l|l|}
\hline$x$ & $k=2$ & $k=4$ & $W G M$ & $R H F$ \\
\hline 0 & 1 & 1 & 1 & 1 \\
0.5 & 0.60703 & 0.60700 & 0.607006 & 0.6070341 \\
1 & 0.37451 & 0.37448 & 0.374462 & 0.3744773 \\
1.5 & 0.24175 & 0.24176 & 0.241759 & 0.2417950 \\
2 & 0.15546 & 0.15540 & 0.155404 & 0.1554150 \\
2.5 & 0.089703 & 0.089749 & 0.089745 & 0.0897578 \\
3 & 0.045579 & 0.045576 & 0.045574 & 0.0455772 \\
3.5 & 0.019474 & 0.019444 & 0.019403 & 0.0195095 \\
4 & 0.006392 & 0.006392 & 0.00639265 & 0.00639244 \\
4.5 & 0.001408 & 0.001405 & 0.00140666 & 0.00140578 \\
5 & 0.0001759 & 0.0001756 & 0.000175756 & 0.00017527 \\
5.5 & 0.0000108 & 0.0000103 & 0.0000101114 & 0.00001005 \\
6 & 0.0000002 & 0.0000002 & 0.0000002075 & 0.00000002 \\
\hline
\end{tabular}

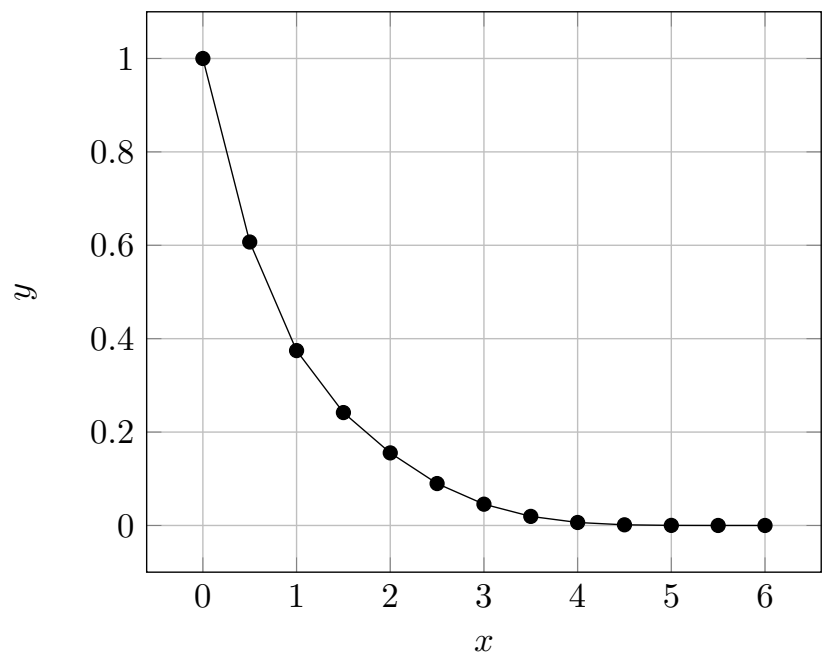

Fig. 2. Discontinuous Galerkin spectral element method solution with $k=4$

the answer in this paper. The advantage of this method is lower computing than using other methods (using fixed matrices) and achieving greater accuracy by increasing the number of elements or degrees of Legendre polynomials.

\section{COMPETING INTEREST}

Author declares that there is no conflict of interest regarding the publication of this paper.

\section{AVAILABILITY OF DATA AND MATERIAL}

The results and numerical data obtained in this paper have been fully tested. These results are obtained using MATLAB R2017a(win64) software and Windows 8 operating system on a intel(R) Core(TM) i $7 \mathrm{CPU}, 1.73 \mathrm{GHz}$ processor with $4 \mathrm{~GB}$ RAM. The authors declare that all data and material in the paper are available and veritable.

\section{REFERENCES}

[1] D. M. Himnelau and K. B. Bischoff, Process Analysis and Simulation, Wiley, New York, 1968.

[2] A. D. Randolph and M. A. Larson, Theory of Particulate Processes, Academic Press, NY, 1971, 01-44, .

[3] P. N. Singh and D. Ramkrishna, Solution of population balance equations by WRM, Comput. Chem. Engng, Vol. 1, 1971, 23-31. 
[4] M. Razzaghi and Y. Ordokhani, An application of rationalized Haar functions to variation problems, Applied Mathematics and Computation, Vol. 22, 2001, 353-64.

[5] A. Alipanah and M. Dehghan, Solution of population balance equations via rationalized Haar functions, Kybernetes 37(8), 2008, 1189-1196

[6] J. J. Reis, R. T. Lynch and J. Butman, Adaptive Haar transformation video bandwith reduction stem for PRVs, Proceeding of the Annual Meeting on Soc. Photo Optic. (San Diego), 1976, 24-35.

[7] P. N. Singh and D. Ramkrishna, Solution of population balance equations by WRM, Comput. Chem. Engng, Vol. 1, 1977, 23-31.

[8] A. D. Randolph, Effect of crystal breakage on crystal size distribution in a mixed suspension crystallizer, I and EC Fund. Math. Anal. Appl, Vol. 8, 1969, 58-63.

[9] C. Hawang and Y. P. Shih, Solution of population balance equations via Block-Pulse functions, J. Chem. Engng, Vol. 25, 1982, 39-45.

[10] R. Y. Chang and M. L. Wang, Shifted Legendre function approximation of differential equations, application to crystalization processes, J. Chem. Engng, Vol. 8, 1984, 117-25.

[11] M. Q. Chen, C. Hwang and Y. P. Shih, A Wavelet-Galerkin method for solving population balance equations, Computers Chem. Engng, Vol. 20 No. 2, 1996, 131-45.

[12] F. B. Hildebrand, Introduction to Numerical Analysis. New York: McGraw-Hill, 1956.

[13] M. Q. Chen, C. Hwang and Y. P. Shih, A wavelet-Galerkin method for solving population balance equations, Computers Chem. Engng., 20(2), 1995, 131-145.

[14] C. Hwang and Y.P. Shih, Solution of population balance equations via Block-pulse functions, J. Chem. Engng., 1982, 39-45.

[15] P. N Singh, D. Ramkrishna, Solution of population balance equations be MWR, Comput. Chem. Engng., 1, 1977, 23-31.

[16] M. Lotfi and A. Alipanah, Legendre spectral element method for solving sine-Gordon equation, Advances in Difference Equations, 119, (2019). DOI 10.1186/s13662-019-2059-7.

[17] J. S. Hesthaven, S. Gottlieb and D. Gottlieb, Spectral Method for TimeDependent Problems, Cambridge University Press, 2007.

[18] C. Pozrikidis, Introduction to Finite and Spectral Element Methods Using Matlab, Chapman and Hall/CRC, 2005. 